\title{
The Effect of Framework Design on Stress Distribution in Implant-Supported FPDs: A 3-D FEM Study
}

\author{
Oguz Eraslan ${ }^{a}$ \\ Ozgur Inan ${ }^{b}$
}

Asli Secilmis ${ }^{c}$

\section{ABSTRACT}

Objectives: The biomechanical behavior of the superstructure plays an important role in the functional longevity of dental implants. However, information about the influence of framework design on stresses transmitted to the implants and supporting tissues is limited. The purpose of this study was to evaluate the effects of framework designs on stress distribution at the supporting bone and supporting implants.

Methods: In this study, the three-dimensional (3D) finite element stress analysis method was used. Three types of 3D mathematical models simulating three different framework designs for implant-supported 3-unit posterior fixed partial dentures were prepared with supporting structures. Convex (1), concave (2), and conventional (3) pontic framework designs were simulated. A 300-N static vertical occlusal load was applied on the node at the center of occlusal surface of the pontic to calculate the stress distributions. As a second condition, frameworks were directly loaded to evaluate the effect of the framework design clearly. The Solidworks/Cosmosworks structural analysis programs were used for finite element modeling/analysis.

Results: The analysis of the von Mises stress values revealed that maximum stress concentrations were located at the loading areas for all models. The pontic side marginal edges of restorations and the necks of implants were other stress concentration regions. There was no clear difference among models when the restorations were loaded at occlusal surfaces. When the veneering porcelain was removed, and load was applied directly to the framework, there was a clear increase in stress concentration with a concave design on supporting implants and bone structure.

Conclusions: The present study showed that the use of a concave design in the pontic frameworks of fixed partial dentures increases the von Mises stress levels on implant abutments and supporting bone structure. However, the veneering porcelain element reduces the effect of the framework and compensates for design weaknesses. (Eur J Dent 2010;4:374-382)

Key words: Framework design; Implant supported fixed partial denture; 3-D FEM; Pontic design.

- associate Professor, University of Selcuk, Faculty of Dentistry, Department of Prosthodontics, Konya, Turkey.

b Professor, University of Selcuk, Faculty of Dentistry, Department of Prosthodontics, Konya, Turkey.

Assistant Professor, University of Gaziantep, Faculty of Dentistry, Department of Prosthodontics, Gaziantep, Turkey.

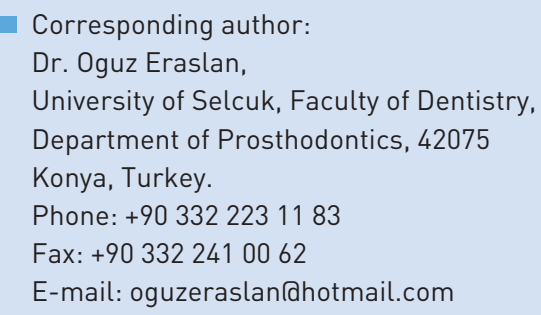




\section{INTRODUCTION}

Recently, all-ceramic fixed partial dentures (FPDs) were introduced; these are currently in use in clinical dental treatment. Due to their superior aesthetics, all-ceramic restorations provide a more desirable approach compared with metal-ceramic combinations for the restoration of natural dentition. ${ }^{1}$ The application of veneering porcelain over a high-strength core ceramic has resulted in the creation of an aesthetically more acceptable laminate composite system, which has been employed in the construction of all-ceramic crowns. ${ }^{2}$ However, ceramics are brittle and susceptible to failure beyond a critical stress, which is dependent on the internal and surface flaw distributions, ${ }^{3,4}$ and their fracture problems have not been solved completely. ${ }^{5}$ Manufacturers have adopted novel engineering processing routes to produce all-ceramic dental core materials with strengths in excess of those achieved using metallic frameworks for metal-ceramic restorations. ${ }^{6}$

The finite element method (FEM) has been shown to be a useful tool when investigating complex systems. ${ }^{7,8}$ Knowledge of stress distribution is important in the understanding of fatigue yielding. ${ }^{9}$ Overall stress distribution within the tooth/ restoration complex is determined by geometry and hard tissue/restorative material arrangement. ${ }^{10}$ It has also been reported that the majority of failures of all-ceramic FPDs originate at the gingival side of the connectors, at the interface of the framework and veneer porcelain. ${ }^{11}$ Thus, the framework design of all-ceramic restorations may have an important effect on stress distribution. Furthermore, the distribution of stress influences the success of the treatment. ${ }^{12-14}$

In recent years, alternative materials and designs have been developed to suit different clinical situations, ${ }^{15}$ and FPDs must have a design type that satisfies certain structural requirements. An FPD must provide enough strength to resist the forces of occlusion that cause flexure of the framework, producing stress in the restoration, and the abutment. It is known that the arch-type design is the most efficient method of forming a structure with materials that have good compressive strength and low tensile strength. ${ }^{16}$ However, to develop theories of prosthesis design, the amount of stress likely to be generated in the oral cavity must be quantified..$^{15}$ Thus, this study aimed at evaluating the effects of framework designs on stress distribution at the supporting bone and supporting implants. The null hypothesis of the current study was that the different framework designs associated with all ceramic restorations would not affect stress distribution.

\section{MATERIAL AND METHODS}

The study was conducted using 3D FEM and the Solidworks 2007 9.0.3 structural analysis program (Solidworks Corporation, USA). A 3D FEM model was constructed to represent a three-unit implant supporting FPDs; this was used to perform the computer simulation (Figure 1a). The model contained a three-unit all-ceramic FPD with two implants (ITI solid screw implants, 3.8-mm diameter, 10-mm bone sink depth; Straumann AG, Waldenburg, Switzerland) at each end as abutment. These were supported by alveolar bone structures simulated as spongy bone surrounded by $2 \mathrm{~mm}$ of cortical bone. Initially, the cross-sections of bone structures included in the mathematical model were hand drawn. They were sketched separately at the front and right planes for each unit in the computer environment. The implant system modeling was conducted using a laser-based 3D scanner (Dental Wings, Montreal, Quebec, Canada) to reproduce the exact dimensions. The implant system was modeled as a single unit with its abutment. The coordinates of the contouring points were then joined to form each structure's volume; together, these defined the final geometry of the FEM model (Figure $1 b-c$ ).

The simulated geometries used for analysis were three different framework designs (convex (1), concave (2), and conventional (3) pontic framework designs) (Figure 2a-c). All-ceramic FPDs (IPS e-max Press; Ivoclar Vivadent AG) were simulated as the restorative material. The geometric models were meshed with tetrahedral quadratic elements (Figure 3a). Each mathematical model included approximately 137,000 nodes and 97,000 solid elements. The bottom exterior nodes of the alveolar bone in the FEM models were fixed in all directions as the boundary condition (Figure 3b). A 300-N static vertical occlusal load ${ }^{17}$ was applied on the node at the center of occlusal surface of the pontic to calculate the stress distributions (Figure 3b). As a second condition, frameworks were di- 
rectly loaded to clearly demonstrate the effect of framework design (Figure 3c).

The materials used in study were assumed to be homogenous and isotropic. The elastic properties of the materials (Young's modulus (E) and Poisson's ratio $(\mu))$ were determined from the literature and are given in Table 1. ${ }^{15,18}$ The FEM modeling was accomplished using the Solidworks software program, and analyses were run with the Cosmosworks software program, which is integrated with Solidworks.

\section{RESULTS}

Results are presented by considering the von Mises criteria. ${ }^{19-24}$ Calculated numerical data were transformed into color graphics to better visualize the mechanical phenomena in the models. Both a 3D whole-model view and mesiodistal cross-sectional views were presented for each condition. All stress values were indicated in mega pascals (MPa). A narrower range was used for the stress indicator scale to better visualize the differences in stress distribution ( 0 to $5 \mathrm{MPa}$ ).

The analysis of the von Mises stress values revealed that maximum stress concentrations were located at the loading areas for all models. In addition, high stress values were located at the gingival embrasures of connectors and the cervical regions on the pontic side of abutment implants in all models. The cervical and inter-implantal alveolar bone structure was the other stress concentration area.

\section{Loading from veneering porcelain}

There were no clear differences in stress distributions among the three design types (Figure $4 a-c)$. The stress trajectory started from the force application point and continued through the framework to the abutment implants, where it was distributed at the bone structure. However, there was intense stress concentrated in ceramic restorations. The highest stress values observed at the force application areas were 27.3, 29.1, and 26.0 MPa for convex, concave, and conventional designs, respectively. The highest value was observed at the veneering porcelain at the force application area of concave design (29.1 MPa). The stress values observed at the cervical regions of the implants were 1.17, 1.41, and $1.35 \mathrm{MPa}$ for convex, concave, and biconcave designs, respectively. The stress values at the alveolar bone structure between implants were 0.31-0.76 MPa (Figure 5a-c). The structures' maximum von Mises stress values with different models are summarized at Table 2 .

\section{Direct loading of framework}

The highest stress values observed at the force application areas of the framework porcelain were $5.12,5.34$, and 5.24 MPa for convex, concave, and biconcave designs, respectively. Although the maximum stress concentration values were close, clear stress distribution diversities were observed between different design types (Figure ba-c). For example, stress values of 4.6-5.34 MPa clearly occupied more space in the concave design framework than the other design types. In addition, high stress concentration values at the implant neck occupied more space in the concave design. The maximum von Mises stress values observed at the cortical bone structure were $0.81,1.01$, and 0.68 MPa for convex, concave, and biconcave designs, respectively. The stress distribution trajectory at the bone structure was different for different designs. While stress was directed toward interimplantal alveolar bone in the concave framework design, at the opposite was the case for the convex and conventional framework designs (Figure $7 a-c)$. However, the maximum stress value of 0.81 MPa was clearly more widespread for the convex design type than for the conventional framework design. The maximum von Mises stress values of structures with different models and a direct loading of the framework are summarized in Table 3.

\section{DISCUSSION}

The stress distribution patterns varied with different framework designs. Based on these results, the null hypothesis that the framework design would not affect the stress distribution for implant-supported FPDs was rejected.

In the present study, using the FEM technique, the effect of framework design on amount and distribution of stress was evaluated. Direct invivo measurement of stress distribution for these materials is difficult. However, a theoretical and well-known method for calculating stress distribution within complex structures is FEM, which allows the investigator to evaluate the influence of model parameter variation once the basic model has been correctly defined. ${ }^{8}$ 


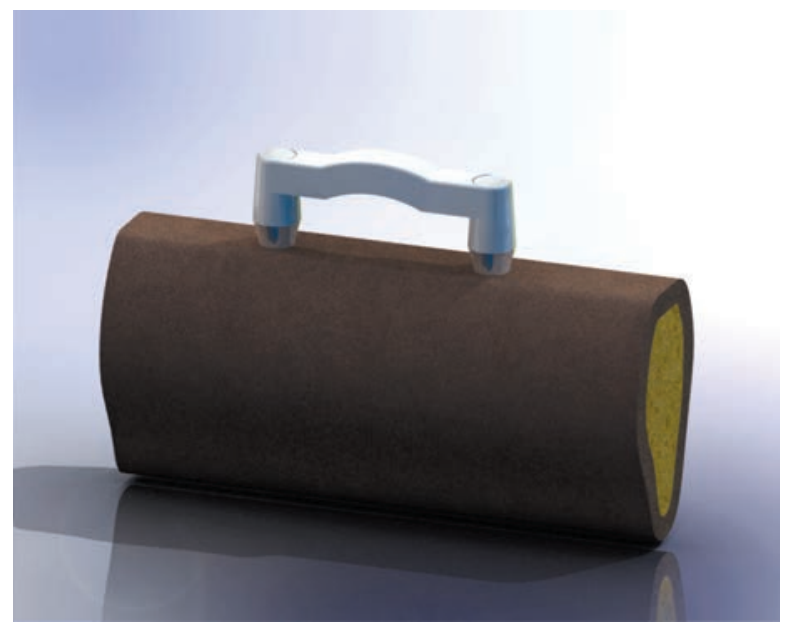

Figure 1a. Rendered view of 3D FEM model simulation of a three-unit implant-supported FPD framework.

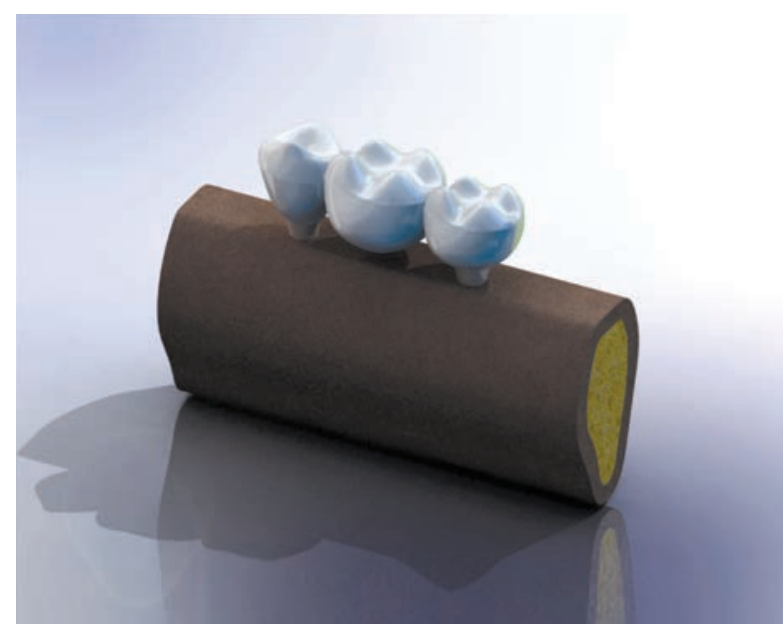

Figure 1b. 3D FE model used in the study with structures rendered according to simulated units.
The model used in this study involved several assumptions regarding simulated structures. The structures in the model were all assumed to be homogeneous, isotropic, and linearly elastic. The properties of the materials modeled in this study, however, and particularly the living tissues, are different. In addition, it is important to point out that the stress distribution patterns may have been different depending on the materials and properties assigned to each layer of the model, as well as the model used in the experiments. ${ }^{15}$ Furthermore, only a vertical loading condition was used to compare different designs. Horizontal or oblique force applications will be used in future

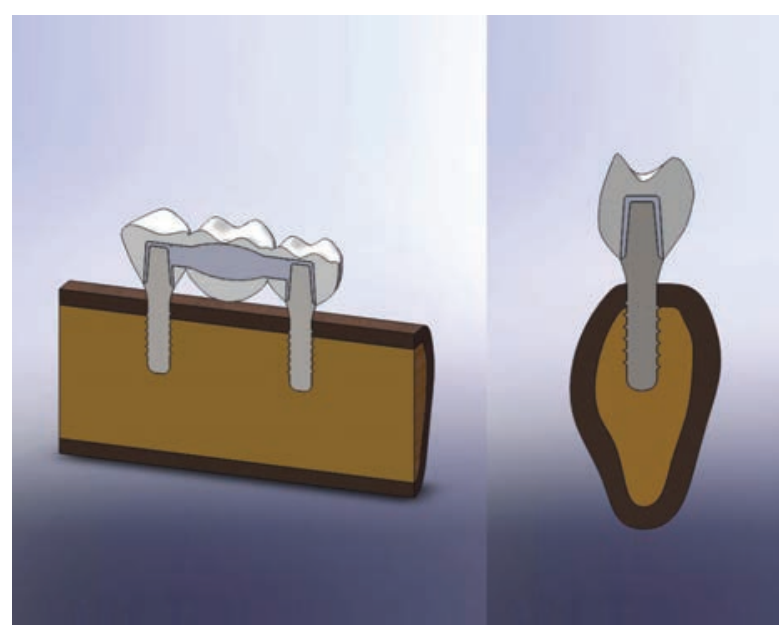

Figure 1c. Mesiodistal and buccolingual cross-section of 3D FEM model and its substructures.

Table 1. Mechanical properties of investigated materials (*acquired from manufacturer).

\begin{tabular}{lcc}
\hline Material & Elastic Modulus (E) (GPa) & Poisson's Ratio (ц) \\
\hline IPS e.max core* & 95 & 0.24 \\
IPS e.max veneer* & 65 & 0.24 \\
Titanium18 & 110 & 0.35 \\
Cortical bone15 & 13.7 & 0.3 \\
Spongy bone15 & 1.37 & 0.3 \\
\hline
\end{tabular}

Table 2. The maximum von Mises stress values of structures involved in different models for force application from veneering porcelain (MPa).

\begin{tabular}{lccccc}
\hline Design\Material & Veneer & Framework & Implant & Cortical bone & Spongy bone \\
\hline Convex & 27.3 & 7.09 & 1.17 & 0.76 & 0.31 \\
Concave & 29.1 & 7.92 & 1.41 & 0.76 & 0.31 \\
Biconvex & 26 & 7.26 & 1.35 & 0.72 & 0.33 \\
\hline
\end{tabular}

Table 3. The maximum von Mises stress values of structures involved in different models for force application from framework porcelain (MPa).

\begin{tabular}{lccccc}
\hline Design\Material & Veneer & Framework & Implant & Cortical bone & Spongy bone \\
\hline Convex & - & 5.12 & 2.28 & 0.81 & 0.35 \\
Concave & - & 5.34 & 2.32 & 1.01 & 0.39 \\
Biconvex & - & 5.24 & 1.66 & 0.68 & 0.34 \\
\hline
\end{tabular}


studies. Thus, the inherent limitations in this study should be considered.

The FEM results are presented in terms of the von Mises stress values. Von Mises stresses depend on the entire stress field and are a widely used indicator of the possibility of damage occurring. ${ }^{20}$ The value of the occlusal force was selected to be $300 \mathrm{~N}$. However, it is not necessary for this force to match its real value exactly because standardization between conditions has been ensured

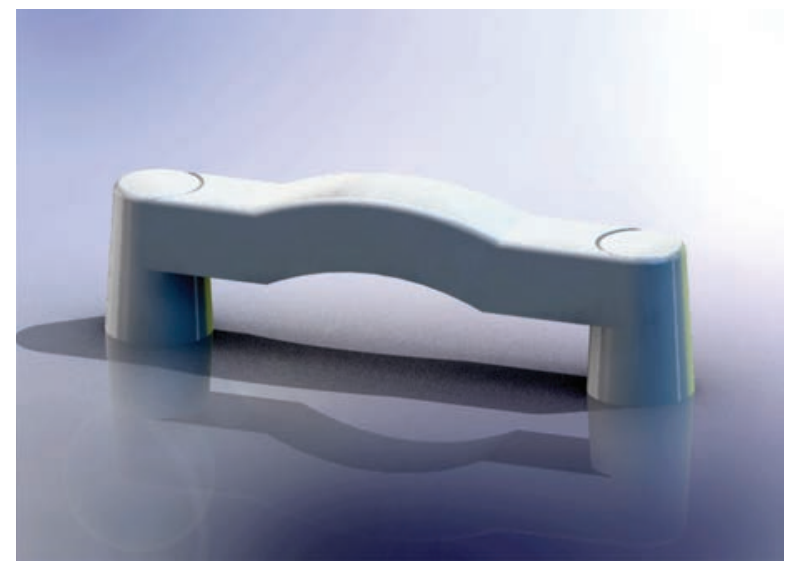

Figure 2a. Illustration of convex pontic framework design

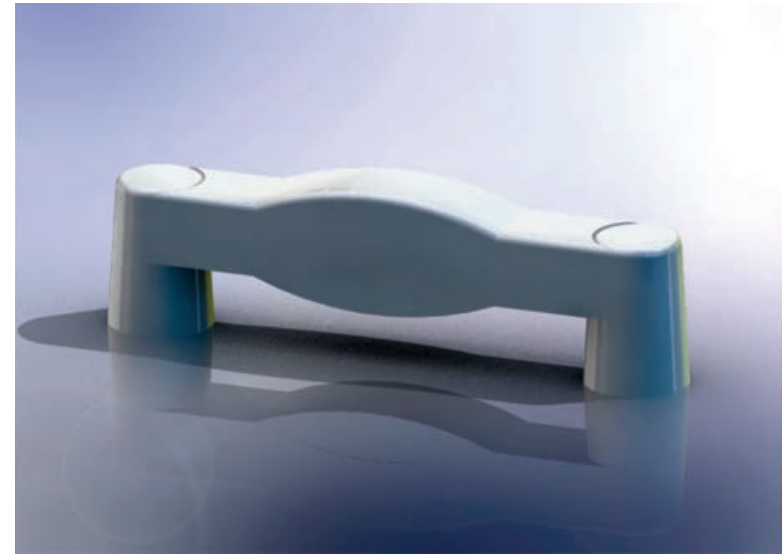

Figure 2c. Illustration of biconvex pontic framework design.

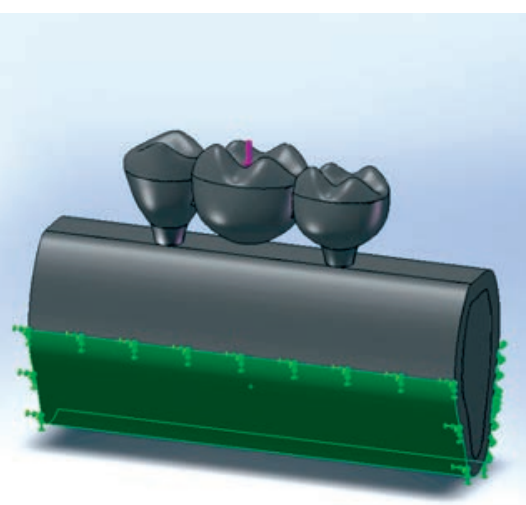

Figure 3b. Load application and boundary condition of veneering porcelain. The pink arrow represents the load application, and the green area is assumed to be fixed as a boundary condition. in the current study and the conditions have been compared qualitatively with one another..$^{15}$ Chen and $\mathrm{Xu}^{7}$ emphasized that the value of FEM modeling has to do with relative values calculated for distribution patterns.

A comparison of stress distributions with different design types revealed lower stress values in frameworks with the convex design. The stress levels for the conventional design type were close to those of the convex design, but the maximum

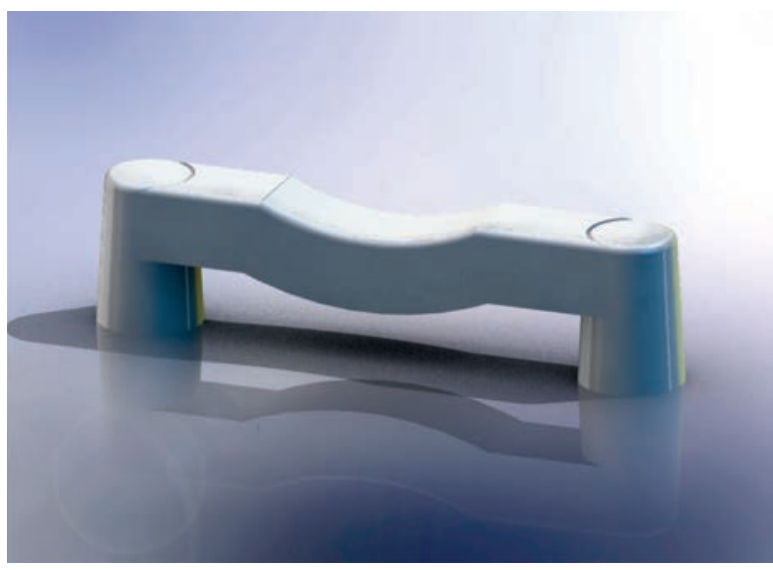

Figure $2 \mathrm{~b}$. Illustration of concave pontic framework design.

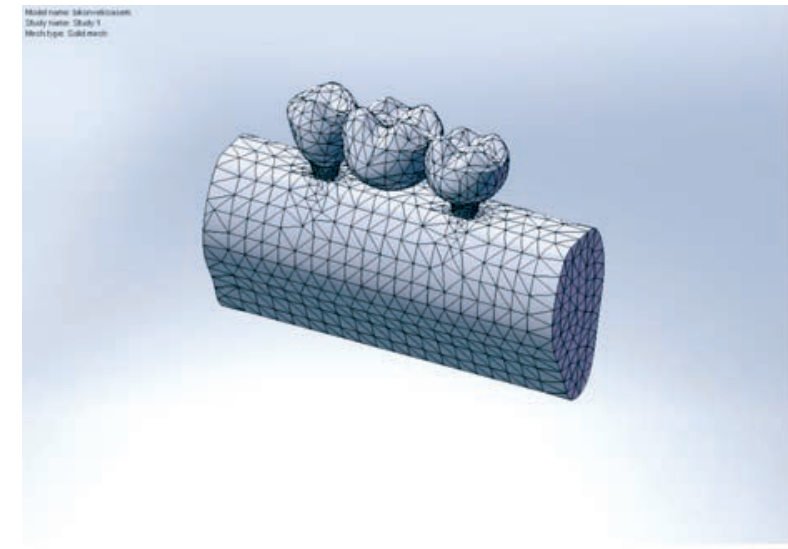

Figure 3a. Geometric model meshed with tetrahedral quadratic elements.

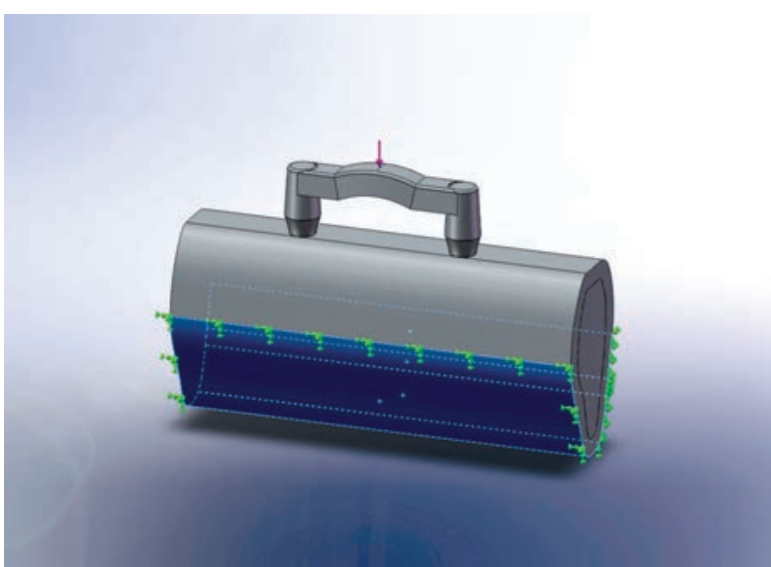

Figure 3c. Load application and boundary condition of direct loading of the framework. The pink arrow represents the load application, and the blue area is assumed to be fixed as a boundary condition. 
stress value occurred at more places in the framework structure with the conventional type of design. Thus, it may be concluded that, biomechanically, the more favorable framework design was the convex one. It was also seen that the veneering porcelain part of the restorations compensated for the mechanical effects of different framework designs. However, more clinical and

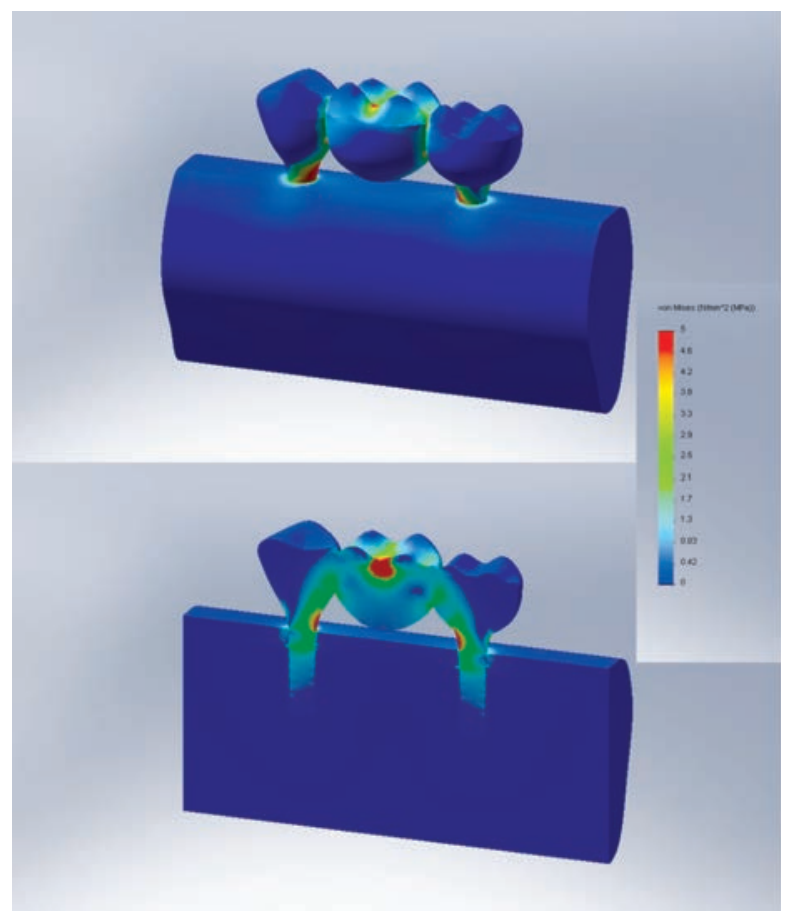

Figure 4a. Distribution of von Mises stresses ( $\mathrm{MPa}$ ) in loading from veneering porcelain with a convex design. Views of main model and its mesiodistal cross-section. Blue to red colors represent lower to higher stress values.

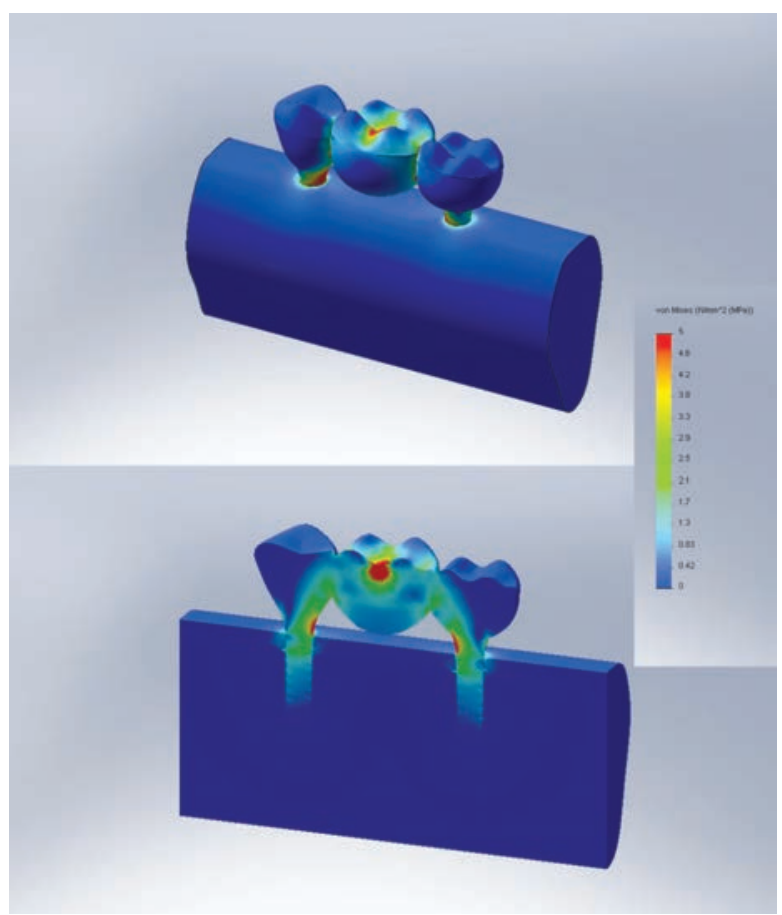

Figure 4b. Distribution of von Mises stresses (MPa) in loading from veneering porcelain with a concave design. Views of main model and its mesiodistal cross-section. experimental studies are needed to support the findings of the current study.

Occlusal loads are transmitted from the point of force application to the point of retainers and, ultimately, travel through the abutments to the bone structure. Moreover, the purpose of a restoration is to maintain strength while resisting the forces of occlusion. Thus, fixed partial dentures must include materials and a design type that provides enough strength to resist the forces of occlusion. Porcelain has an amorphous structure and this produces physical properties typical of a glass. ${ }^{4}$ When porcelain is stressed in tension, small flaws tend to open up and propagate, resulting in a low tensile strength. ${ }^{4}$ However, porcelain is much stronger in terms of compression, because compressive stresses tend to close up flaws. ${ }^{4}$ It is known that when a beam is loaded, the applied loads have a tendency to cause failure of the beam in two main ways: by shearing or by bending the beam. ${ }^{16}$ Arches are commonly used in several structural forms and represent the most efficient method of forming a structure with materials that have good compressive strength and low tensile strength. ${ }^{16}$ Dental porcelains are among such materials because of the abovementioned properties. In order to achieve the most efficient structural solution, The design engineer (in this case, the dentist), can alter the structural configu-

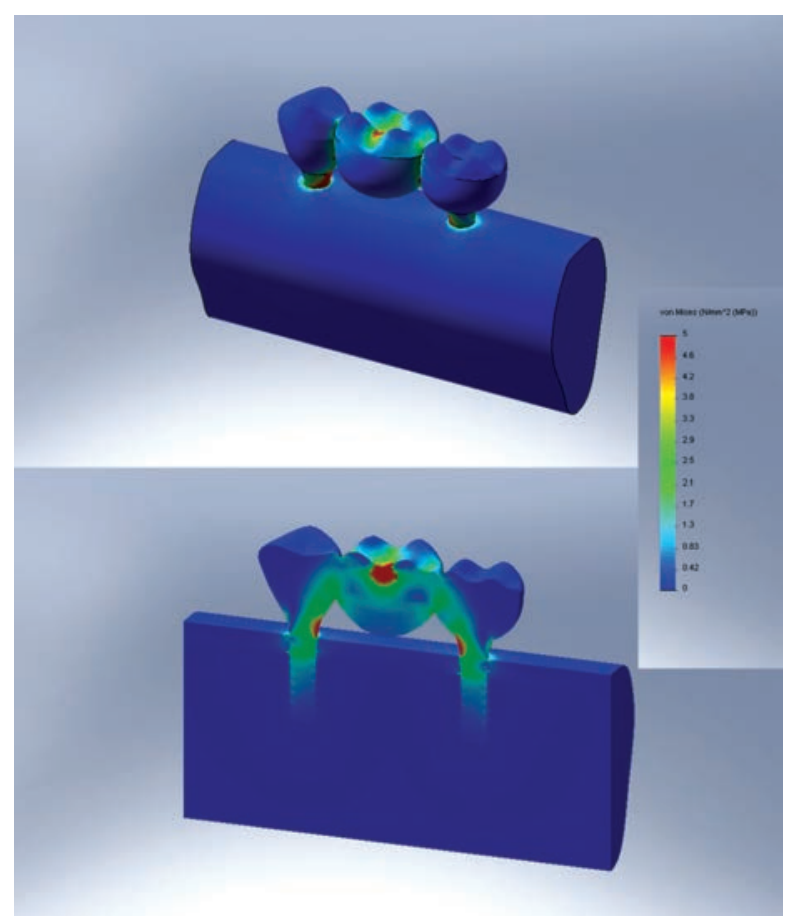

Figure 4c. Distribution of von Mises stresses (MPa) in loading from veneering porcelain with a biconvex design. Views of main model and its mesiodistal cross-section. 
ration to produce an equilibrium condition made up of compression forces. ${ }^{16}$ Thus, the convex framework design can be the most suitable way for inserting ceramic FPDs to restore a missing tooth or teeth. In agreement with this information, the results of the current study revealed that von

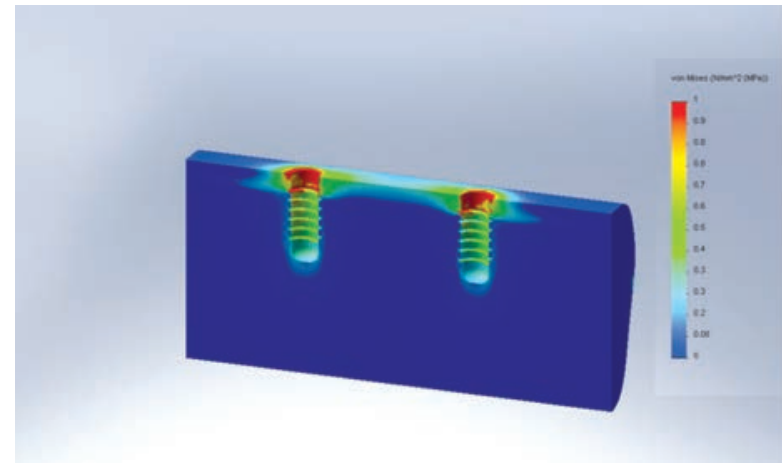

Figure 5a. Distribution of von Mises stresses (MPa) at the bone structure, loading from veneering porcelain with a convex design.

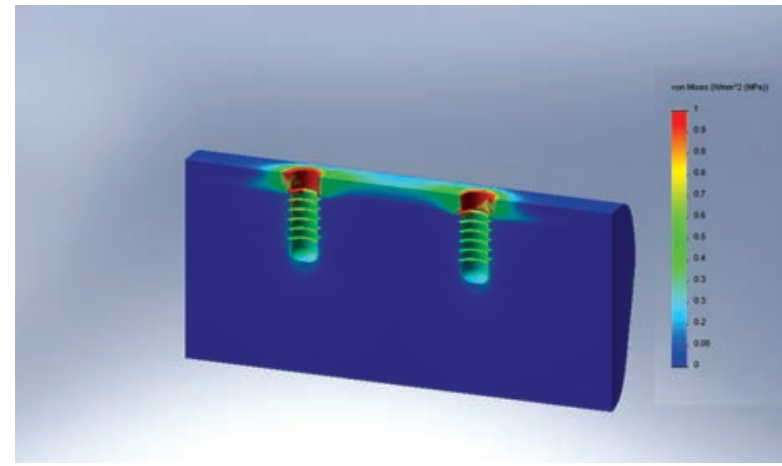

Figure $5 \mathrm{~b}$. Distribution of von Mises stresses (MPa) at the bone structure, loading from veneering porcelain with a concave design.

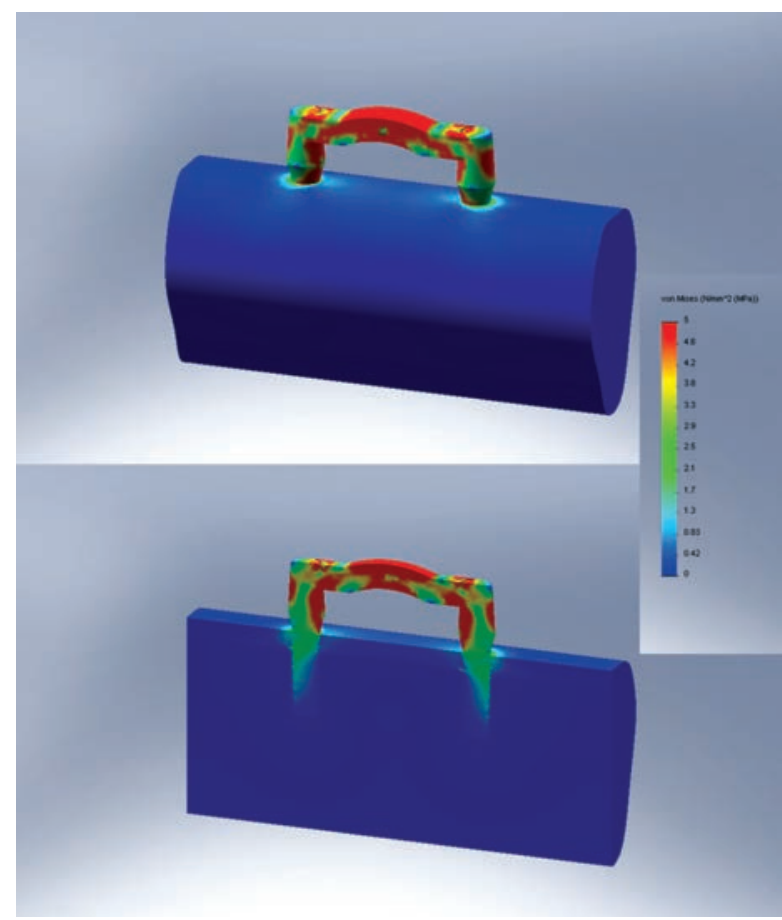

Figure 6a. Distribution of von Mises stresses (MPa) through direct loading of the framework with a convex design. Views of main model and its mesiodistal crosssection.
Mises stress values decreased with the use of a convex design type.

When dealing with implant-supported FPDs, modifying the framework design may help to decrease stress concentrations, but the veneering porcelain element may reduce the effect of a framework with recent restorative materials. Further studies that better simulate the oral environment and including fatigue loading are recommended. Although the clinical implications that can be derived from this study are important, long term clinical evidence is required.

\section{CONCLUSIONS}

Within the limitations of this study, the following conclusions were drawn:

- The use of a concave design for FPD pontic

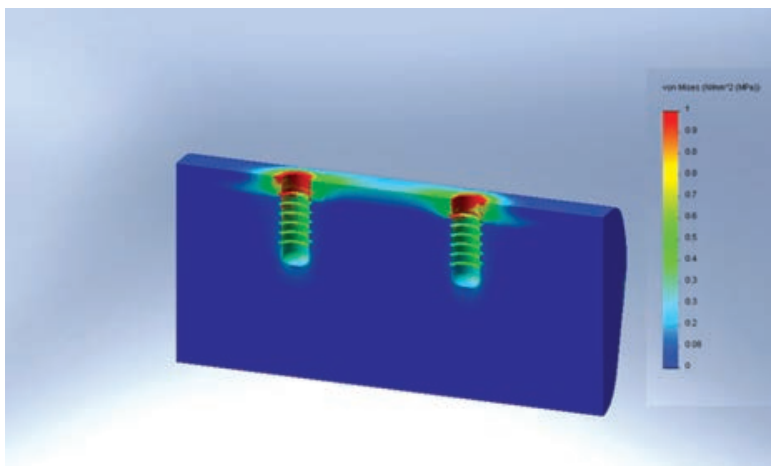

Figure 5c. Distribution of von Mises stresses (MPa) at the bone structure, loading from veneering porcelain with a biconvex design.

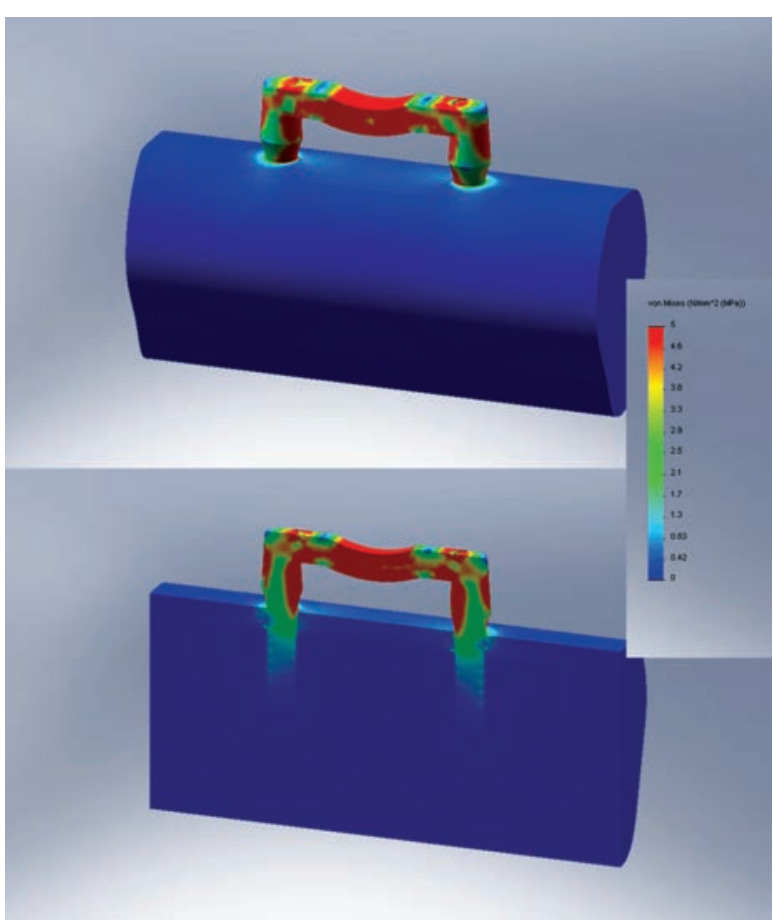

Figure 6b. Distribution of von Mises stresses (MPa) through direct loading of the framework with a concave design. Views of main model and its mesiodistal crosssection 


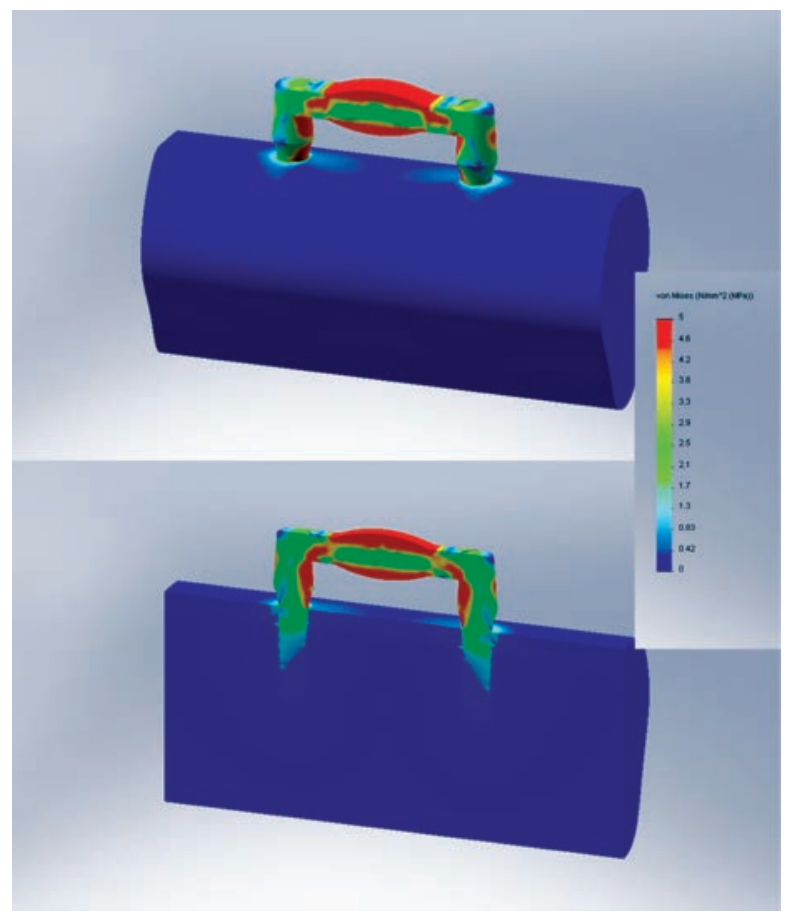

Figure 6c. Distribution of von Mises stresses (MPa) through direct loading of the framework with a biconvex design. Views of main model and its mesiodistal crosssection.

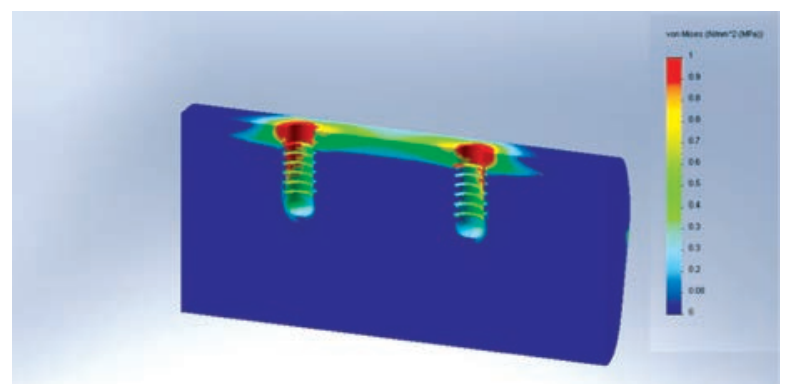

Figure 7a. Distribution of von Mises stresses (MPa) at the bone structure through direct loading of the framework with a convex design.

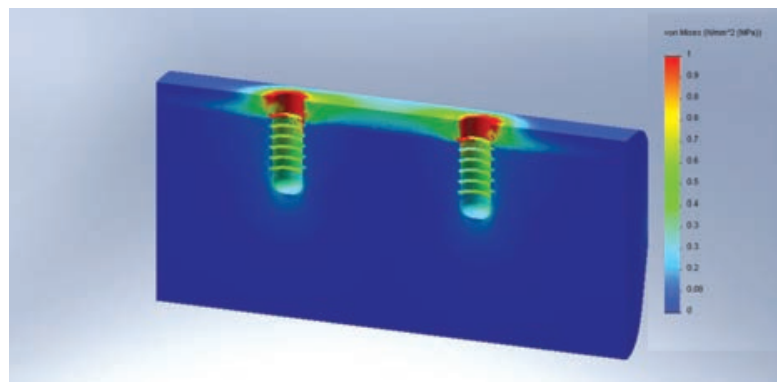

Figure 7b. Distribution of von Mises stresses (MPa) at the bone structure through direct loading of framework with a concave design.

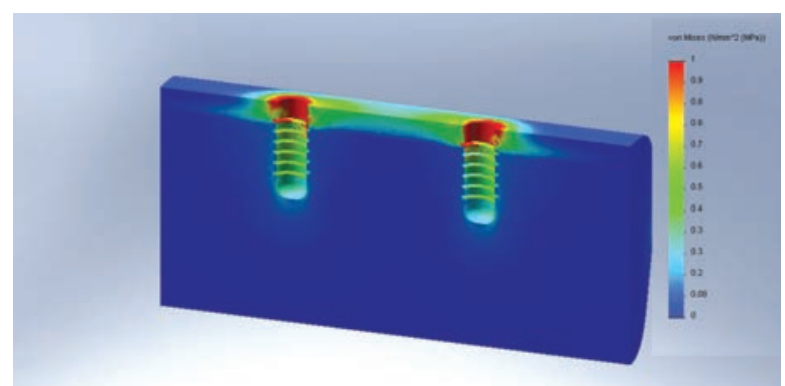

Figure 7c. Distribution of von Mises stresses (MPa) at the bone structure through direct loading of the framework with a concave design. frameworks increases the von Mises stress levels on implant abutments and supporting bone structure.

- The veneering porcelain part reduces the effect of the framework and compensates for design weaknesses.

\section{ACKNOWLEDGEMENT}

This study is funded by Research Projects Council of the University of Selcuk.

\section{REFERENCES}

1. Burke FJ, Qualtrough AJ, Hale RW. Dentin-bonded all-ceramic crowns: current status. J Am Dent Assoc 1998;129:455-460.

2. Fleming GJ, Nolan L, Harris JJ. The in-vitro clinical failure of all-ceramic crowns and the connector area of fixed partial dentures: the influence of interfacial surface roughness. J Dent 2005;33:405-412.

3. Kingery WD, Bowen HK, Uhlmann DR. Elasticity, anelasticity, and strength. In: Kingery WD, editor. In: Introduction to ceramics. New York: Wiley; 1976:768-805.

4. O' Brien WJ. Dental Materials and their selection, 2nd ed. Chicago: Quintessence Pub. Co, Inc., 1997:287-302.

5. Tsumita M, Kokubo Y, Ohtsuka T. Influences of core frame design on the mechanical strength of posterior all-ceramic fixed partial dentures. Part 1. Two dimensional finite element analyses. Tsurumi University Dental Journal 2005;31:205-212.

6. Fleming GJ, Dickens M, Thomas LJ, Harris JJ. The in vitro failure of all-ceramic crowns and the connector area of fixed partial dentures using bilayered ceramic specimens: the influence of core to dentin thickness ratio. Dent Mater 2006;22:771-777.

7. Chen J, Xu L. A finite element analysis of the human temporomandibular joint. J Biomech Eng 1994;116:401-407.

8. Lanza A, Aversa R, Rengo S, Apicella D, Apicella A. 3D FEA of cemented steel, glass and carbon posts in a maxillary incisor. Dent Mater 2005;21:709-715.

9. Magne P, Perakis N. Stress distribution of inlay-anchored adhesive fixed partial dentures: A finite element analysis of the influence of restorative materials and abutment preparation design. J Prosthet Dent 2002;87:516-527.

10. Magne $P$, Versluis A, Douglas WH. Rationalization of incisor shape: experimental-numerical analysis. $J$ Prosthet Dent 1999;81:345-355.

11. Kelly JR, Tesk JA. Sorensen JA. Failure of all ceramic fixed partial dentures in vitro and in vivo: analysis and modeling. J Dent Res 1995;74:1253-1258. 
12. Decock V, De Nayer K, De Boever JA, Dent M. 18-year longitudinal study of cantilevered fixed restorations. Int $J$ Prosthodont 1996;9:331-340.

13. Goodacre CJ, Kan JK, Rungcharassaeng K. Clinical complications of osseointegrated implants. J Prosthet Dent 1999;81:537-552.

14. Williams KR, Watson CJ, Murphy WM, Scott J, Gregory $M$, Sinobad D. Finite element analysis of fixed prostheses attached to osseointegrated implants. Quintessence Int 1990;21:563-570.

15. Eraslan O, Sevimay M, Usumez A, Eskitascıō̄lu G. Effects of cantilever design and material on stress distribution in fixed partial dentures - a finite element analysis. J Oral Rehab 2005;32:273-278.

16. Nageim H Al, Durka F, Morgan W, Williams D. Structural mechanics loads, analysis, design and, materials. 6th ed. Malaysia: Pearson Edu. Ltd., 2003;144-308.

17. Eskitascioglu G, Usumez A, Sevimay M, Soykan E, Unsal $E$. The influence of occlusal loading location on stresses transferred to implant-supported prostheses and supporting bone: A three dimensional finite element study. J Prosthet Dent 2004;91:144-150.

18. Sevimay M, Usumez A, Eskitascioglu G. The influence of various occlusal materials on stresses transferred to implant-supported prostheses and supporting bone: a threedimensional finite-element study. J Biomed Mater Res B Appl Biomater 2005;73:140-147.

19. Beer FP, DeWolf JT, Johnston ER. Mechanics of materials. 4th ed. Singapore: McGraw-Hill Int, 2005;360-378.

20. Pegoretti A, Fambri L, Zappini G, Bianchetti M. Finite element analysis of a glass fibre reinforced composite endodontic post. Biomaterials 2002;23:2667-2682.

21. Akça K, Iplikçioḡlu H. Finite element stress analysis of the influence of staggered versus straight placement of dental implants. Int J Oral Maxillofac Implants 2001;16:722-730.

22. Timoshenko S, Young DH. Elements of strength of materials. 5th ed. Florence: Wadsworth, 1968:377.

23. Ugural AC, Fenster SK. Advanced strength and applied elasticity. 4th ed. New York: Prentice-Hall, 2003:155-157.

24. Yang HS, Lang LA, Molina A, Felton DA. The effects of dowel design and load direction on dowel-and-core restorations. J Prosthet Dent 2001;85:558-567. 